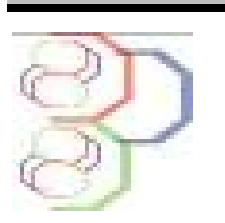

Journal of Applied Biosciences 106:10224 -10235

ISSN 1997-5902

\title{
Nomenclature vernaculaire et diversité des variétés locales des gombos (Abelmoschus spp.) cultivées au Bénin
}

\author{
Gnawe M. ${ }^{1}$, Yedomonhan H. ${ }^{1}$, Adomou A. C. ${ }^{1}$, Houenon H. ${ }^{1}$, Dansi A. ${ }^{2}$, Akoegninou A. ${ }^{1}$ \\ 1 : Laboratoire de Botanique et Écologie Végétale (LaBEV), Faculté des Sciences et Techniques, Université \\ d'Abomey-Calavi, 01 BP 4521 Cotonou (Bénin). \\ 2 : Laboratoire de Biotechnologies, Ressources Génétiques et Amélioration des Espèces Animales et Végétales \\ (BIORAVE), Faculté des Sciences et Techniques de Dassa, Université Polytechnique d'Abomey, BP 14 Dassa \\ (Bénin). \\ * : Auteur correspondant : gnamac2002@yahoo.fr
}

Original submitted in on $17^{\text {th }}$ June 2016. Published online at www.m.elewa.org on $31^{\text {st }}$ October 2016

http://dx.doi.org/10.4314/iab.v106i1.2

\section{RESUME}

Objectif : L'objectif de ce travail est de recenser toutes les variétés locales de gombos cultivées au Bénin en vue de déterminer leurs caractéristiques agro-morphologiques.

Méthodologie et Résultat : L'étude a été réalisée par le biais d'enquêtes ethnobotaniques auprès de 760 producteurs de gombos à travers 31 ethnies. Au total, 24 noms vernaculaires et 480 appellations sont utilisés pour désigner respectivement le mot gombo et ses variétés locales. La nomenclature populaire ne fait aucune distinction entre les différentes espèces existantes et repose sur l'utilisation des caractères morphologique, agronomique et culturel. Le nombre de variétés locales recensées varie de 3 chez les boufalé à 41 chez les bariba, avec un cumul de 387 variétés locales pour le Bénin.

Conclusion et application : Le nombre de variétés locales cultivées par producteur varie de 1 à 6 . II n'est statistiquement ni lié à la profession des producteurs $(p=0,919)$, ni lié à leur âge $(p=0,173)$. Par contre, il varie significativement en fonction du sexe $(p=0,048)$ et de l'ethnie $(p=0,000)$. La connaissance de la diversité variétale réelle au plan national nécessite la caractérisation agro-morphologique des variétés locales recensées afin de connaître celles qui ont des noms synonymes ou homonymes.

Mots clés. Gombo, variétés locales, parataxonomie, diversité, ethnie, Bénin

\begin{abstract}
Objective: The objective of this work is to identify all the local varieties of okra grown in Benin to determine their agro-morphological characteristics.

Methodology and Results : The study was conducted through ethnobotanical surveys of 760 producers okra through 31 races. A total of 24 and 480 common names and designations are used to designate the word gumbo and local varieties respectively. The popular nomenclature makes no distinction between existing species and based on the use of morphological characters, agronomic and cultural. The number of local varieties identified from 3 to 41 in Boufale in bariba, with a total of 387 local varieties for Benin.
\end{abstract}


Conclusion and Application :The number of local varieties grown by the producer from 1 to 6 . It is not statistically related to the profession of producers $(p=0.919)$ nor linked to age $(p=0.173)$. By cons, it varies significantly by gender $(p=0.048)$ and ethnicity $(p=0.000)$. Knowing the actual varietal diversity at the national level requires the agro-morphological characterization identified local varieties to find those synonyms or homonyms names.

Keywords: Okra, local varieties parataxonomy, diversity, ethnicity, Benin

\section{INTRODUCTION}

Les gombos appartiennent au genre Abelmoschus, à la famille des Malvaceae, à l'ordre des Malvales, à la sous-classe des Dillenidae, à la classe des Magnoliopsida et à la division des Magnoliophyta (Spichiger et al., 2000). Ils se répartissent en 7 espèces dont $A$. angulosus, $A$. callei, A. crinitus, $A$. esculentus, $A$. ficulneus, $A$. manihot et $A$. moschatus (Hamon et Charrier, 1997). Parmi ces espèces de gombos, 4 sont cultivées (A. callei, A. esculentus, $A$. manihot et $A$. moschatus) et les 3 autres ( $A$. angulosus, $A$. crinitus et $A$. ficulneus) sont strictement spontanées (Borssum-Waalkes, 1966). Au Bénin, les gombos cultivés (Abelmoschus spp.) constituent l'un des légumes hautement prisés. En effet, leurs feuilles et fruits sont consommés par tous les groupes ethniques sous forme de sauce gluante (Dansi, 2008 ; Achigan-Dako, 2010). Cependant, leur culture est relativement très faible et ne fournit que 42.816 tonnes/an pour une superficie moyenne de 13.272 ha/an, soit un rendement de 3,23 tonnes/ha (FAOSTAT, 2013). Cette production ne représente que $4,04 \%$ de la production du Nigéria qui est le premier pays producteur du gombo en Afrique d'une part et $0,53 \%$ de la production mondiale d'autre part. De plus, le rendement de production du Bénin reste nettement inférieur à celui de 7,57 tonnes/hectare au plan mondial. Par ailleurs, la culture de gombos au Bénin est assujettie au régime pluviométrique. Ainsi, les gombos sont cultivés surtout en saison pluvieuse et exceptionnellement en saison sèche le long des cours d'eau plus ou moins importants (Dansi, 2008;

\section{MÉTHODE D'ÉTUDE}

Milieu d'étude : Le Bénin est situé entre $6^{\circ} 15^{\prime}$ et $12^{\circ} 25^{\prime}$ de latitude nord et entre $0^{\circ} 40^{\prime}$ et $3^{\circ} 45^{\prime}$ de longitude est. D'une superficie de $112.600 \mathrm{~km}^{2}$, il est limité au nord par le Niger, au sud par l'Océan Atlantique, à l'est par le Nigéria et à l'ouest par le Togo et le Burkina-Faso
Achigan-Dako, 2010) du fait de leur forte sensibilité au déficit hydrique du sol (Nana et al., 2010). La conséquence est que leur prix moyen passe de 0,6 FCFA/fruit en saison pluvieuse (juin-novembre) à 25 FCFA/fruit en saison sèche (décembre-mai) par suite de la baisse drastique du ratio offre/demande. Cette augmentation de la valeur marchande des gombos semble être au-delà de l'économie des ménages ruraux dont le revenu mensuel est de 6.171 à 10.454 FCFA (INSAE, 2013). Ceci constitue une menace à la sécurité alimentaire au Bénin. Pourtant, l'Agenda $21 \mathrm{du}$ Gouvernement béninois a souligné la nécessité d'asseoir la sécurité alimentaire, gage de tout développement socio-économique. II est donc nécessaire de pallier à la faible production et au faible rendement de production des gombos au Bénin afin de contribuer à leur promotion pour la sécurité alimentaire et la réduction de la pauvreté. De nombreux travaux portant sur la taxonomie, la phylogénie, la répartition géographique, les caractéristiques botaniques et écologiques, les propriétés chimiques et pharmaceutiques et les usages alimentaires ont été réalisés sur les gombos cultivés et leur synthèse est présentée par Siemonsma et Kouamé (2004). Au Bénin, les travaux réalisés sur les gombos restent encore partiels et n'ont été consacrés qu'à l'étude de la diversité et de l'ethnobotanique des gombos dans le sud-ouest du pays (Gnawé, 2015). L'objectif de cette étude est de recenser toutes les variétés locales de gombos cultivées sur toute l'étendue du pays.

(Akoègninou et al., 2006). La pluviosité est comprise entre 900 et $1400 \mathrm{~mm}$ d'eau par an avec un gradient croissant ouest-est et nord-sud (Akoègninou et al., 2006). La répartition de la pluie dans l'année permet de distinguer trois grands types de climat dans le pays : le 
climat tropical humide ou subéquatorial au sud, le climat tropical humide de transition au centre et le climat tropical sec au nord. Sur le plan pédologique, la nature de la roche mère, le niveau de la nappe phréatique et la topographie du milieu déterminent deux grands types de sols. Le premier type est constitué des sols drainés comprenant les sols ferralitiques, les sols ferrugineux, concrétionnés ou non, avec ou sans cuirasses, les sols minéraux bruts et les sols bruns eutrophes. Le second type regroupe les sols hydromorphes à hydromorphie temporaire ou permanente dont les vertisols et les sols halomorphes (Akoègninou et al., 2006). Le réseau hydrographique comprend deux groupes de cours d'eau qui s'écoulent dans deux directions principales (Akoègninou et al., 2006). Le premier groupe comprend les fleuves Mono et Ouémé qui se jettent dans l'Océan Atlantique au sud. Le second groupe regroupe les rivières Sota, Alibori, Mékrou qui se jettent dans le fleuve Niger au nord. Dans le sud du pays, les cordons littoraux créent un important complexe de lacs (le lac Ahémé et le lac Nokoué) et de lagunes dont la lagune côtière et celle de Porto-Novo. Sur le plan démographique, le Bénin abrite 9.983.884 habitants, avec un taux annuel de croissance de $3,51 \%$ et une densité de la population de 87 habitants/km² (INSAE, 2013). Cette population s'exprime en 60 langues regroupées en 9 groupes sociolinguistiques dont les fon et leurs apparentés (39\%), les adja $(15 \%)$, les yoruba $(12 \%)$, les bariba $(9 \%)$, les peulh $(7 \%)$, les ottamari $(6 \%)$, les yoa-lokpa $(4 \%)$, les dendi $(3 \%)$ et les autres (5\%) (Amadou et Atodjinou, 2012). L'agriculture est la principale activité réalisée et occupe $49 \%$ des populations (INSAE, 2013). Elle est suivie du commerce $(27 \%)$ et de l'artisanat $(17 \%)$.

Collecte des données: Le choix des villages et producteurs de gombos a été fait avec l'aide des Techniciens Spécialisés en Production Végétale (TSPV) des Centres d'Actions Régionaux pour le Développement Rural (CARDER) qui sont les techniciens agricoles étatiques ayant pour rôle d'appuyer les producteurs dans leurs activités agricoles. Au total, 760 producteurs de gombos répartis dans 129 villages (figure 1) ont été enquêtés. Ils se répartissent dans 31 ethnies et sont en majorité des illettrés $(70,92 \%)$. Les enquêtés sont à $51,05 \%$ des hommes et à $48,95 \%$ des femmes. Les enquêtes ont eu lieu par des interviews individuelles à l'aide d'un questionnaire semi-structuré. Les rubriques dudit questionnaire sont les noms vernaculaires des espèces et variétés locales cultivées avec leur étymologie et leur origine.

Traitement des données : La liste exhaustive en nom vernaculaire des espèces et variétés locales de gombos cultivées a été établie. La diversité variétale a été évaluée à l'échelle des producteurs par le biais du nombre de variétés locales cultivées puis à l'échelle des ethnies par le biais du nombre de variétés citées, de l'indice de diversité de Shannon et de l'équitabilité de Piélou. L'indice de diversité de Shannon $(\mathrm{H})$ a été calculé suivant la formule de Frontier et Pichod-Viale (1995) : $\mathrm{H}=$ $\Sigma\left(n_{j} / N\right) \ln \left(n_{i} / N\right)$, où $n_{i}$ est le nombre de citation de la variété $i$ par les producteurs d'une ethnie donnée et $N$ est la somme des $n_{i}$ pour l'ensemble des variétés citées pour l'ethnie considérée. La diversité est faible lorsque $\mathrm{H}<3$ bits, moyenne si $\mathrm{H}$ est compris entre 3 et 4 puis élevée quand $H \geq 4$ bits. L'indice d'équitabilité ( $E$ ) a été déterminé suivant la formule : $E: H / l n(R)$, avec $H$ : l'indice de diversité de Shannon et $R$ : le nombre total de variétés citées. II varie 0 à 1 . Plus, il tend vers 1 , plus il y a une citation équitable des variétés au sein de l'ethnie. L'Analyse de Variance (ANOVA) a été réalisée pour vérifier la variabilité de la diversité variétale au sein des groupes socioprofessionnels. Le test de chi-2 a été réalisé pour voir la dépendance ou non entre le nombre de variétés locales citées par ethnie et la taille de l'échantillon enquêté. Le logiciel utilisé est MINITAB 14.0. Le statut de production de chacune des variétés locales au sein des ethnies a été déterminé sur la base des fréquences de citation. Ainsi, 4 classes de variétés locales ont été définies par ethnie, à savoir : les variétés très populaires (variétés citées par $75 \%$ des producteurs de l'ethnie considérée), les variétés populaires (fréquence de citation comprise entre 50 et $75 \%$ ), les variétés peu populaires (fréquence de citation comprise entre 25 et $50 \%$ ) puis les variétés rares (fréquence de citation est inférieure à $25 \%$ ). 


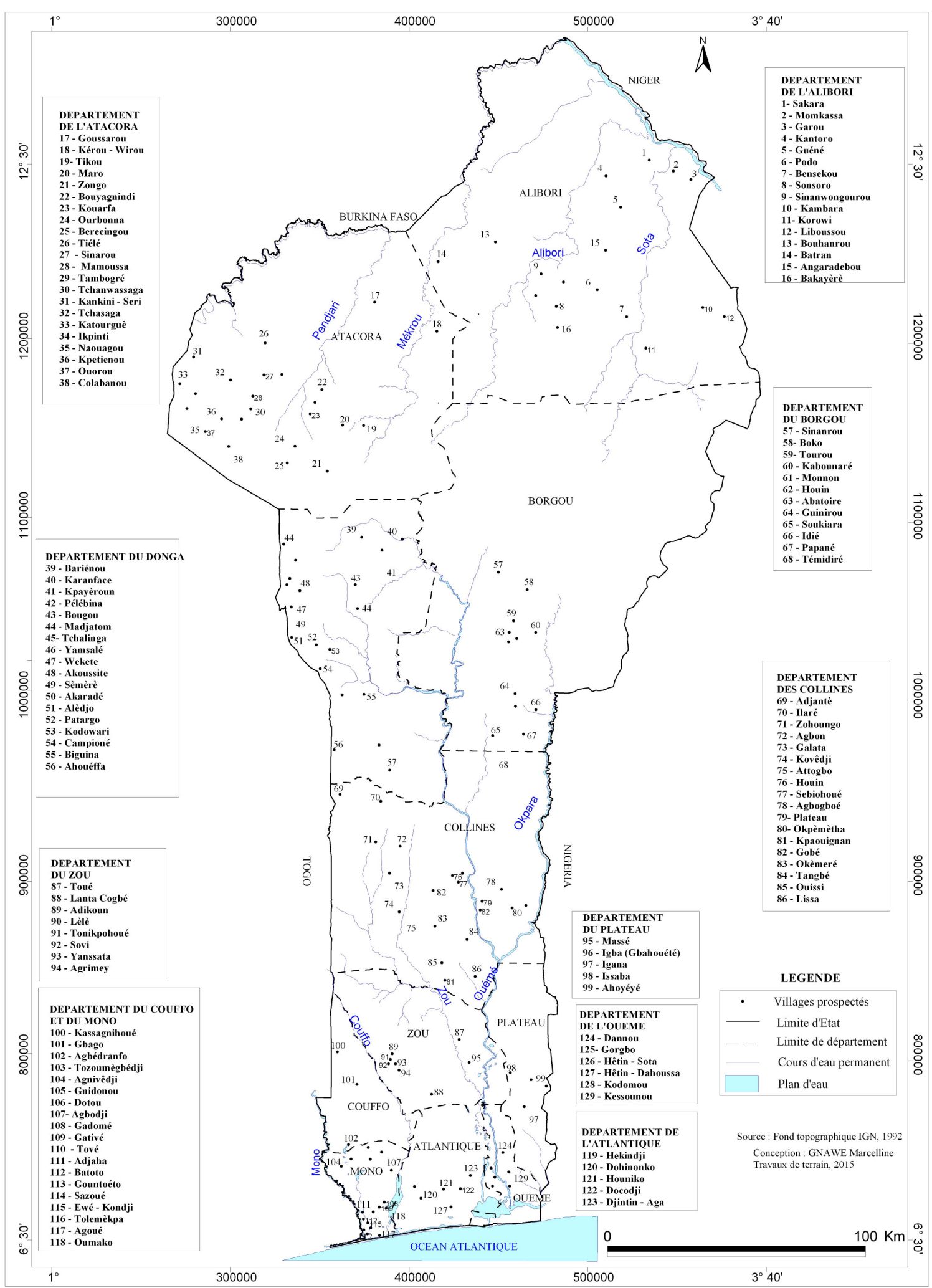

Figure 1 : Carte du Bénin montrant la localisation géographique des villages explorés 


\section{RÉSULTAT}

Nomenclature vernaculaire des différentes espèces de gombos: Aucun des producteurs enquêté n'a reconnu différemment les espèces cultivées. Les gombos, considérés au rang spécifique, ont les mêmes appellations vernaculaires dans une même langue locale. Au total, 24 appellations ont été recensées comme noms désignant le mot gombo (tableau 1). Parmi elles, 4 ont des synonymes comme dinouri qui est le singulier de yênouri (au pluriel) en langue ditamari, yêbonou et kpéwogo qui sont synonymes de yabonou en langue bariba, la qui est synonyme de laafé en dendi puis kènouêkê qui est le singulier de sinouanssi (au pluriel) en m'bermè. Les noms vernaculaires du mot gombo varient en fonction des aires socioculturelles et même des ethnies au sein de la même aire socioculturelle. Dans ce dernier cas, il s'agit par exemple de l'aire socioculturelle Adja chez qui les gombos sont appelés êfé en Aïzo, fétri en Mina, ninhoun en Sahouè et Kotafon puis nonnou en Mahi. Sur les 9 groupes socioculturels, seules les aires socioculturelles dendi et peulhs, représentées chacune par une seule ethnie, n'ont pas connu de variation de noms vernaculaires pour les gombos.

Tableau 1 : Appellations en langues locales des espèces de gombos cultivées

\begin{tabular}{|c|c|c|c|}
\hline $\mathrm{N}^{\circ}$ d'ordre & Noms vernaculaires & $\begin{array}{l}\text { Langues locales } \\
\text { (ethnies) }\end{array}$ & $\begin{array}{c}\text { Aires socioculturelles des } \\
\text { ethnies }\end{array}$ \\
\hline 1 & dinouri $^{*}$ ou yênourè ${ }^{\star *}$ & Ditamari & Ottamari \\
\hline 2 & êfé & Aïzo & Adja \\
\hline 3 & fétri & Adja, Mina, Ouatchi, Xwla & Adja \\
\hline 4 & févi & Fon, Wémè & Adja \\
\hline 5 & ikounou & Ani & Yoa-Lokpa \\
\hline 6 & ila & $\begin{array}{c}\text { Idaasha, Ifè, Holli, Koura, } \\
\text { Nagot, Tchabè }\end{array}$ & Yoruba \\
\hline 7 & kpé & Boo & Bariba \\
\hline 8 & la, laafé & Dendi & Dendi \\
\hline 9 & laardjè & Peulh & Peulh \\
\hline 10 & lakouan & Bialé & Ottamari \\
\hline 11 & mannan & $\begin{array}{c}\text { Boufalé, Kotokoli, Lokpa, } \\
\text { Yoom, }\end{array}$ & Yoa-Lokpa \\
\hline 12 & manya & Waama & Ottamari \\
\hline 13 & ninhoun & Kotafon, Sahouè & Adja \\
\hline 14 & nonnou & Mahi & Adja \\
\hline 15 & quékpina & Foodo & Yoa-Lokpa \\
\hline 16 & worko & Mokolé & Yoruba \\
\hline 17 & yabonou, yêbonou, kpéwogo & Bariba & Bariba \\
\hline 19 & Sinouanssi**, kènouêkê ${ }^{*}$ & M'bermè & Ottamari \\
\hline
\end{tabular}

${ }^{*}$ : au singulier, ${ }^{* *}$ : au pluriel

Étymologie des noms et critères de nomenclature des variétales locales : Au total, 480 appellations ont été recensées pour les différentes variétés locales de gombos (Abelmoschus spp.) cultivées à travers les 31 ethnies enquêtées au Bénin (tableau 2). Les noms vernaculaires des variétés locales de gombos sont, dans $47,50 \%$ des appellations, constitués de 2 mots dont le nom générique attribué au mot gombo précédé ou suivi d'un nom ou d'un adjectif relevant le caractère distinctif de la variété. Dans les $52,50 \%$ restants des appellations, les noms des variétés locales sont dépourvus des noms attribués indifféremment aux espèces de gombos. Ces noms sont des noms, des adjectifs ou des groupes de mots faisant appel à des caractères propres aux variétés au Bénin. En tenant compte des caractères utilisés pour nommer les différentes variétés locales de gombos, 9 caractères ont été recensés (figure 2). Parmi ces derniers, la forme du fruit et la durée nécessaire pour la floraison (durée du cycle végétatif) transparaissent respectivement dans $36,83 \%$ et $35,25 \%$ des noms des variétés locales et représentent alors les caractères les plus utilisés dans la nomenclature vernaculaire des gombos. Les caractères comme la ressemblance à d'autres fruits $(1,44 \%)$, la valeur culinaire $(1,29 \%)$ et 
l'allusion aux interdits alimentaires $(1,15 \%)$ sont utilisés dans une moindre mesure. Parmi les appellations recensées, $1,63 \%$ dérivent de la combinaison de 3 caractères et $38,29 \%$ sont issues de l'usage simultané de
2 caractères. Les $60,29 \%$ d'appellations restants reposent exclusivement sur un seul caractère.

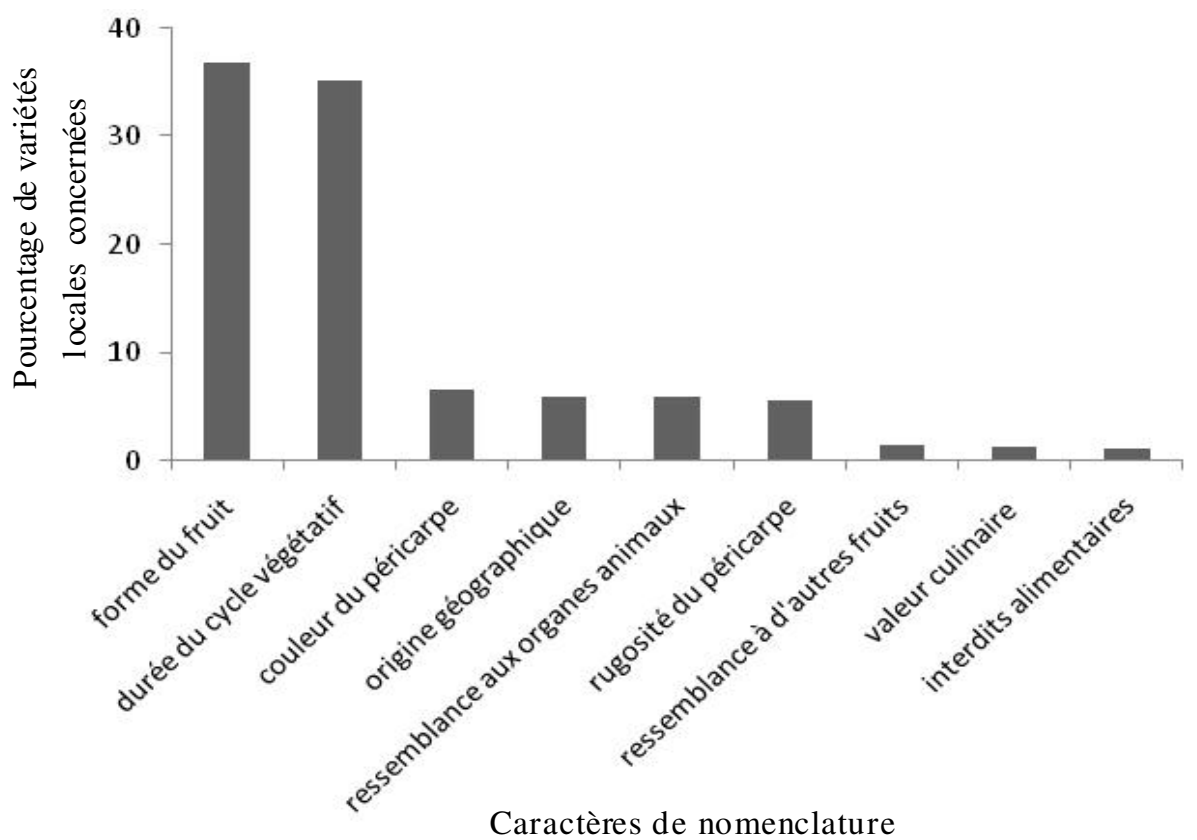

Figure 2 : Fréquence relative des caractères de nomenclature des variétés locales de gombos cultivées

Tableau 2 : Listes des variétés locales de gombo recensées par ethnie avec leur statut de production

\begin{tabular}{|c|c|}
\hline Ethnie & Noms vernaculaires et statut des variétés locales \\
\hline adja & $\begin{array}{l}\text { chouathonkoun (wlélétchiamèthon, houétchiamèthonfétri, tchéotafétri, houétchithon, houétrithonfétri), } \\
\text { efanfétri, éhouéfétri (gbotrou), éhouizo (houizo), essofétri, gogolécomè, goninie, hidamandji, } \\
\text { houéchivéfétri (houétchiamèvéfétri, houétrivéfétri, houétchivé, wlélétchivéfétri, wlélétchiamèvéfétri) (+), } \\
\text { kegnanou, kpédévinonvo, kpégono, n'kégbanfétri (wlélétchidékafétri, houétchidéka), sidanon, sinkoun }\end{array}$ \\
\hline aïzo & éféga( $( \pm)$, éfégloui(+), éféhouédékpo (éhouéfé) (+), éfésouanwéda \\
\hline ani & $\begin{array}{l}\text { atchéléiloor, ekouloufornon, koudjouloukou(+), mansalaka(++), mansalaka-akétréka, mansalakakalata, } \\
\text { mansalakalamenta }\end{array}$ \\
\hline bariba & $\begin{array}{l}\text { agamanaki, bibamanlé, bordé, courtoubi, dèmousakamèra, ganganhoun, kouhounto-kouhoutosouanrou, } \\
\text { kpahakoba, kpahakobi pidoue, kpahakobi (kpassiroukobi, kpassiboukora), kpassakobi (kpassikobi), } \\
\text { kpéhoubourougui, kpésséroukora (wokéroukora), kpéworgodindèman (yâbodidinnou), kpéworgokpidoue, } \\
\text { malanvikpé, malanvikpéboussirou, marékpé, monré, sonhonkou yâbonou, sonkoumarosso, } \\
\text { sonsarérouyâbonou, souroutia, sourouyirou souanrou (worgosouanrou), tchouboudoukpé } \\
\text { (woubourouyâbonou), trouwoukérou, wékérokora, wirouboka, wiroubortaré, worgokouhouto- } \\
\text { kouhoutopiga, worgosouanroutia, worgotoégourou, yâbipikounou, yâbo fannou, yaboguénon, } \\
\text { yâbohoukounou, yabokpégué, yabonou orgossou (sonkoumanragou, orokonou, sigiworou, sikiworou)( } \pm \text { ), } \\
\text { yabonourokonou, yabopipinou, yayaré }\end{array}$ \\
\hline bialé & $\begin{array}{l}\text { kakouandaà, koukoulakouna, lakoualaa, lakouan sibouhoun, lakouandaà, lakouandaàlognéni, } \\
\text { lakouandaàmanké, lakouanlàà( } \pm \text {, lakouannii, lakouannirou, lakoubiara, lakoudakouannan (lakouannian), } \\
\text { lapohoun, lognénoun, lopéréchéti, nainnii (lakouandaàtchitchéé) }\end{array}$ \\
\hline
\end{tabular}




\begin{tabular}{|c|c|}
\hline \multicolumn{2}{|c|}{$\begin{array}{l}\text { Gnawe et al. J. Appl. Biosci. } 2016 \quad \text { Nomenclature vernaculaire et diversité des variétés locales des } \\
\text { gombos (Abelmoschus spp.) cultivées au Bénin }\end{array}$} \\
\hline Ethnie & Noms vernaculaires et statut des variétés locales \\
\hline boo & $\begin{array}{l}\text { bouzièkourouna, bouzièkpé (kpébouziè), da-kassa, gbéssihin, gbéssiman, kouloumi, kpassabèna, } \\
\text { kpassaè, kpéguéssénan, kpékouloumi, kpékpassakoba (bouzièagbana, bouzièkpéogban, } \\
\text { bouzièguéssénan), kpélèdé, kpéobénà, kpéortonantian, kpéssaè }\end{array}$ \\
\hline boufalé & flannanmaanan( $( \pm)$, koumoloukou(+), mantéléka(++) \\
\hline dendi & $\begin{array}{l}\text { gandibior, guérilii (géri-hili, lafé jéri-hili) (+), handouhikaïzé, handouhizaïzé, kitigbo, la éroko, la } \\
\text { fronlonkou, lafé kaïdjikpè (kongouwa), lafé koiré, lafé kparè, lafébi idoubor, lafébi ikoukou, lafébior, } \\
\text { lafécaïdjikoè, laféi ikoukou, lagnénon, lagoonan, lambou-lafé, méléman }\end{array}$ \\
\hline ditamari & $\begin{array}{l}\text { bounouyar (ekakonoura), dipacoyênoura (dipanoura), inanboanou, inouda( } \pm \text { ), inoudaï, inoudayé, inouhoï } \\
\text { (inouhoï-ihani), inouni, inoupia (inoupiè, tounoukpêti), inoussaï (inoudà yênourè), nignonnoura, } \\
\text { tinoussorti, typèignéhiiti, yêckanourê, yênoubouhoukèyê, yênoudaryè, yênoukouffa, } \\
\text { yênouranignonkpara( } \pm \text { ) (yêkpakonoura, ikakonou), yênousso, yênouworgo }\end{array}$ \\
\hline fon & $\begin{array}{l}\text { adjafévi, adjakpanon, agbanlifévi, ahoudidi, aloufévi (houéfévi, alouloufévi)( } \pm \text { ), atakou, azangbanfévi } \\
\text { (févisouzandokpo), cassato, corbia, dohoukpa, féviadjahênain, féviahoudidi, févigaga (aloga, finkoun), } \\
\text { févigli, févisouzandokpoadadé, féviyaougnon, féviyaya, gbannanmèfévi, kpassafévi, lanzo (aganlizo, } \\
\text { agbozo), mahufévi, sinkou, souanthonfévi (souanthonmêthon), souanwéninhoun (azanquandéko, } \\
\text { souzanwéninnoun, souwémèfévi), tchohubor, yodana, zidognonou (honnon, zikponon) }\end{array}$ \\
\hline foodo & $\begin{array}{l}\text { atchangbélésséé, dépalquékpinan, doubouré-tégbélou, kabouassi, koumolouor( } \pm \text { ), mansalaka, } \\
\text { tchanmanni }\end{array}$ \\
\hline holli & ila bilè, ila elènouhêyê, ila iroko, ila ochoumêdji-abor, ila ohouya, ila olikpankou, ila olochoumêtha(+) \\
\hline idaatcha & $\begin{array}{l}\text { ila aladjaka, ila alaritan, ila coutonou, ila éko, ila èlègougou, ila érébété, ila foufou, ila gougou, ila } \\
\text { icoutonou, ila idatcha, ila idéti éwou (idéti éwou), ila odjo, ila olochoumèdji, ila olochoumèroun, ila } \\
\text { olochoumètha, ila olochoumèthagougou, ila olochoumèthakékéré, ila olochou-obou (ila ordjorgban)( } \pm \text { ), ila } \\
\text { olokpichoblé( } \pm \text {, ila oroun }\end{array}$ \\
\hline ifê & $\begin{array}{l}\text { iho agnongni (ila iwoagbanli)( } \pm \text { ), ila adja, ila ado, ila agbanli, ila foufou (oko gnibo), ila icoutonou, ila iroko } \\
\text { (ila oroun)(+), ila iwougolo (iwougolo)( } \pm \text { ), ila kaboli, ila mansalaka, ila odjo, ila oko, ila olochoumèdji, ila } \\
\text { sôtème, kobéréroun }\end{array}$ \\
\hline kotafon & רouéninhoun, gbotrou, gotordoui, houéninhoun, houizo, kegnann \\
\hline kotokoli & koudjouloukou(+), maanan kébéza, mansalaka(++) \\
\hline koura & $\begin{array}{l}\text { borthélébor, ignélaffa, ila dichoux (koudjouloukou)( } \pm \text { ), ila dodjo, ila doubou(+), ila foufou, ila kékéré, ila } \\
\text { kokoro(+), ila kpikpan, mansalaka }\end{array}$ \\
\hline lokpa & $\begin{array}{l}\text { djanmaanin (koumolou, koumourou, koumouroukou)(+), koumorouhu, kpaamannan, kulaalèè, maanan } \\
\text { coutarè, maanan coutobléé, maanan kulaalèè, manhuhloman, mansola( }(+) \text {, mansola-sosowa, mantélaga, } \\
\text { mantéran, m'goukeyaatô, nanttélo, nonkunmaanan, samanssoumaanan, sélékamaanan, yolouman } \\
\text { maanan, youbissi }\end{array}$ \\
\hline mahi & $\begin{array}{l}\text { adihoumian, adjaka (adjakassi), adjakassigondonhin, adjathon, agbanlizo( } \pm \text { ), atakou, azangbangbé } \\
\text { (féviyaougnon)( } \pm \text { ), azikponon }(z i k p o)( \pm), \text { coutonouninhoun, éhouizo, lougou, sinkou, souanthonthon, } \\
\text { tchohubor, zophou (aloufévi) }\end{array}$ \\
\hline trmè & $\begin{array}{l}\text { icorrégnéni, inongnè, inonni (tinonniité, sikoukounouassi) (++), kannonnirguè, kannougnarguè, kanonnii, } \\
\text { koukounouwonssi, sininmonissi, sinongnassi, sinonniissi, sinonpienssi, tinongnartè }( \pm)\end{array}$ \\
\hline mina & orgnifétri, gbogboloulou, kpèdévifétri(+), vodougbé( \pm ) \\
\hline mokolé & adjòchidji (amantê) (+), amantêsissofoufou, kitigbo(+), kpin-agné( \pm ), kpinayê, yassayà \\
\hline nago & $\begin{array}{l}\text { assomanmy, bamysingnin, ewéaguidi, ibilè, igbéti éwou, ila a } \\
\text { idalèkomandou, ila iho, ila iroko, ila iwo, ila iyé, ila otê( } \pm \text {, ila ov }\end{array}$ \\
\hline peulh & $\begin{array}{l}\text { lardjè bonerdjè douhoundjè goudè, lardjè dabounerdjè (lardjè tchéoudjè, lardjè gnanierdjè)( } \pm \text { ), lardjè } \\
\text { damonerdjè, lardjè danerdjè, lardjè danierdjè douhoundjè jourdè, lardjè douhoundjè lébidiidjè, lardjè } \\
\text { doumouhuondjè, lardjè galargile jarbarrè, lardjè kaéladjè (lardjè-danierdjè-douhourdjè), lardjè-danierdjè- } \\
\text { douhourdjè-goudjè, lardjè-danierdjè-goudjè }\end{array}$ \\
\hline
\end{tabular}




\begin{tabular}{|c|c|}
\hline \multicolumn{2}{|c|}{$\begin{array}{l}\text { Gnawe et al. J. Appl. Biosci. } 2016 \quad \text { Nomenclature vernaculaire et diversité des variétés locales des } \\
\text { gombos (Abelmoschus spp.) cultivées au Bénin }\end{array}$} \\
\hline Ethnie & Noms vernaculaires et statut des variétés locales \\
\hline sahouè & $\begin{array}{l}\text { adjaninhoun (adolph)(+), babadanou, corgboé, dékanvi, égbazinmè, fanwé, gotordoui, houéninhoun, } \\
\text { kétédé, kohounki, sinkoun, souanthonhoun, souzanwéninhoun, tivi( } \pm \text { ), védézoué }\end{array}$ \\
\hline tchabè & abidikpoto, érébété(++), ila foufou, ila ochou-bou( \pm ), youbissi \\
\hline waama & $\begin{array}{l}\text { maanii (wétohina) }(++) \text {, maanitou, mandaafa, mandaatou }( \pm) \text {, mandaya }( \pm) \text {, mannitou, manyapènoutia } \\
\text { (youcamanféya) }\end{array}$ \\
\hline ouatchi & ahouéfétri( \pm ), corgnifétri, kpédévifétri(+), vodougbé, wétrithonfétri \\
\hline wémè & $\begin{array}{l}\text { atchobor (tchorbor)(+), avagbodjifévi, azikpononfévi (févigogonon, féviazikponon) (++), féviahounnon, } \\
\text { fiomèthonfévi, gogononvorvor, gorgbofévikoun, houégbéfévi, massahouéfévi, waouwa }\end{array}$ \\
\hline xhla & ahouéninhoun $(+)$, gbogboloulou, gotordoui, kpédévifétri(++) \\
\hline yoom & $\begin{array}{l}\text { bejeanmononhan, boudjanmaanan }( \pm) \text {, ipèmaanmn, kangnéran (kangnignéro, gorgaséré, kangnéro)( } \pm \text { ), } \\
\text { kapigninou, kpaamaanan }(+) \text {, maanan-mandjèssi, manpiro, waoduhin (kpornoogon), yahomaanan }( \pm) \text {, } \\
\text { youroubissi }\end{array}$ \\
\hline
\end{tabular}

$(++$ : Variétés très populaires (fréquence de citation comprise entre $75-100 \%),+:$ variétés populaires (fréquence de citation comprise entre 50 et $75 \%$ ), \pm : variétés peu populaires (fréquence de citation comprise entre $0-25 \%$ ). Les noms non suivis de signes sont ceux des variétés rares (fréquence de citation inférieure à $25 \%$ ). Les noms entre parenthèses sont des noms synonymes à celui qu'ils succèdent)

Diversité variétale : Au total, 387 variétés ont été recensées auprès des 31 ethnies enquêtées sous réserve des synonymies et homonymies inter-ethniques (tableau 2). Parmi ces variétés recensées, 6 sont très populaires dans leurs 6 ethnies respectives de production. II s'agit des variétés mansalaka les ani et les kotokoli, matéléka chez les boufalé, inonnii (tinonniité ou sikoukounouassi) chez les m'bermè, érébété chez les tchabè, maanii (wétothina) chez les waama, azikpononfévi (févigogonon ou févizikponon) chez les wémè et kpédévininhoun chez les xhla. La classe des variétés populaires et celle des variétés peu populaires comportent respectivement 12 et 21 variétés sous réserve des synonymies et homonymies inter-ethniques. Les 346 variétés restantes, soit $89,94 \%$ de la diversité variété identifiée, sont cultivées par moins de $25 \%$ des producteurs dans les aires ethniques respectives de production et sont alors des variétés peu populaires. A l'échelle des producteurs, le nombre de variétés locales cultivées par producteur varie de 1 à 6 , avec une moyenne de 1,98 $\pm 0,967$ pour la zone d'étude (tableau 3). C'est parmi les producteurs bariba et dendi que certains détiennent individuellement la diversité variétale maximale ( 6 variétés). Par contre, les producteurs xhla et tchabè enregistrent individuellement les plus faibles nombres de variétés (1 à 2). Dans l'ensemble, la diversité variétale détenue par producteur est très faible. La majorité des producteurs ne cultive qu'une seule $(34,34 \%)$ à deux variétés locales $(43,16 \%)$ chacun. Les producteurs cultivant plus de 2 variétés locales ne font que $22,49 \%$ de l'échantillon enquêté dont $15,13 \%$ détiennent 3 variétés locales, $4,47 \%$ disposent de 4 variétés locales, $2,50 \%$ de 5 variétés locales et $0,39 \%$ de
6 variétés locales. Le nombre de variétés locales cultivées par producteur n'est statistiquement ni lié à la profession des producteurs $(p=0,919)$, ni lié à leur âge $(p=0,173)$. Par contre, il varie significativement d'un sexe à l'autre $(p=0,048)$ et d'une ethnie à l'autre $(p=$ $0,000)$. Le nombre moyen de variétés locales détenues par l'homme $(2,04 \pm 1,00)$ est significativement supérieur à celui cultivé par la femme $(1,90 \pm 0,91)$. Quant à l'ethnie, les plus fortes valeurs moyennes de diversité variétale par producteur sont obtenues chez les m'bermè $(2,81 \pm 0,75$ variétés locales), les yoom $(2,71 \pm 0,99$ variétés locales), les biali ( $2,62 \pm 1,20$ variétés locales) et les bariba $(2,56 \pm 1,24$ variétés locales) (tableau 3$)$. Par contre, les plus faibles valeurs moyennes de diversité variétale par producteur sont enregistrées chez les tchabè (1,36 $\pm 0,50$ variétés locales) et les holli (1,45 \pm 0,93 variétés locales). A l'échelle des ethnies, le nombre de variétés locales recensées varie de 3 chez les boufalé à 41 chez les bariba (tableau 3). Le test de chi-2 a révélé l'existence d'une forte dépendance entre le nombre de variétés locales détenues au sein des ethnies et le nombre de personnes enquêtées par ethnie ( $\mathrm{ddl}=30 ; \mathrm{X}^{2}$ $=49,687 ; P=0,013)$. La corrélation de Pearson indique que le nombre de variétés détenues est positivement et significativement corrélé à la taille de l'échantillon enquêté ( $P=0,000 ; r=0,630)$. L'indice de diversité de Shannon a ses fortes valeurs chez les ethnies bariba (3,30 bits) et fon (2,91 bits) puis ses faibles valeurs chez les ethnies xhla (1,13 bit) et boufalé ( 1 bit). II indique que la diversité variétale est partout faible sauf chez les bariba où elle est moyenne. L'équitabilité de Pielou varie de 0,58 chez les wémè à 0,96 chez les aïzo (tableau 3). Sa faible valeur observée chez les wémè traduit la forte 
citation d'une même variété par presque tous les producteurs wémè alors que les autres variétés détenues par la même ethnie ne sont citées que par peu de producteurs. C'est cette même prédominance d'une ou de deux variétés, en termes de fréquence de citation, qui explique aussi les valeurs d'équitabilité relativement faibles observées chez les foodo $(0,65)$, les ani $(0,73)$, les dendi $(0,75)$, les lokpa $(0,76)$, les wachi $(0,76)$, les holli $(0,77)$ et les adja $(0,78)$. Chez les autres ethnies, les valeurs de l'équitabilité de Pielou sont toutes proches de 1 et témoignent de ce que les variétés ont presque les mêmes fréquences de citation au sein de leurs ethnies respectives de production.

Tableau 3 : Variation des paramètres de diversité de gombos cultivés par ethnie

\begin{tabular}{|c|c|c|c|c|c|c|c|}
\hline \multirow[t]{2}{*}{ Ethnie } & \multirow{2}{*}{$\begin{array}{l}\text { Nombre de } \\
\text { personnes } \\
\text { enquêtées }\end{array}$} & \multicolumn{3}{|c|}{$\begin{array}{c}\text { Nombre de variétés locales détenues } \\
\text { par producteur }\end{array}$} & \multirow{2}{*}{$\begin{array}{l}\text { Nombre } \\
\text { total de } \\
\text { variétés } \\
\text { locales }\end{array}$} & \multirow{2}{*}{$\begin{array}{l}\text { Indice de } \\
\text { Shannon } \\
\text { (bits }\end{array}$} & \multirow[t]{2}{*}{$\begin{array}{l}\text { Equitabilité de } \\
\text { Pielou }\end{array}$} \\
\hline & & $\begin{array}{l}\text { Nombre } \\
\text { minimal }\end{array}$ & $\begin{array}{l}\text { Nombre } \\
\text { maximal }\end{array}$ & $\begin{array}{l}\text { Nombre } \\
\text { moyen }\end{array}$ & & & \\
\hline adja & 74 & 1 & 4 & $1,89 \pm 0,88$ & 15 & 2,10 & 0,78 \\
\hline aïzo & 9 & 1 & 3 & $1,67 \pm 0,71$ & 4 & 1,33 & 0,96 \\
\hline ani & 13 & 1 & 5 & $2,08 \pm 0,95$ & 7 & 1,44 & 0,73 \\
\hline bariba & 45 & 1 & 6 & $2,56 \pm 1,24$ & 41 & 3,30 & 0,89 \\
\hline bialé & 13 & 1 & 5 & $2,62 \pm 1,20$ & 16 & 2,60 & 0,94 \\
\hline boo & 26 & 1 & 4 & $1,58 \pm 0,70$ & 15 & 2,55 & 0,94 \\
\hline boufalé & 19 & 1 & 3 & $1,84 \pm 0,50$ & 3 & 1,00 & 0,91 \\
\hline dendi & 49 & 1 & 6 & $1,47 \pm 0,82$ & 19 & 2,20 & 0,75 \\
\hline ditamari & 25 & 1 & 5 & $2,24 \pm 1,05$ & 20 & 2,66 & 0,89 \\
\hline fon & 46 & 1 & 5 & $2,07 \pm 1,06$ & 27 & 2,91 & 0,88 \\
\hline foodo & 25 & 1 & 4 & $1,72 \pm 0,79$ & 7 & 1,27 & 0,65 \\
\hline holli & 11 & 1 & 4 & $1,45 \pm 0,93$ & 7 & 1,51 & 0,77 \\
\hline idatcha & 36 & 1 & 4 & $1,72 \pm 0,97$ & 20 & 2,55 & 0,85 \\
\hline ifê & 29 & 1 & 5 & $2,14 \pm 1,03$ & 16 & 2,28 & 0,82 \\
\hline kotafon & 29 & 1 & 5 & $1,55 \pm 0,87$ & 14 & 2,12 & 0,80 \\
\hline kotokoli & 17 & 1 & 4 & $2,13 \pm 1,02$ & 6 & 1,44 & 0,81 \\
\hline koura & 18 & 1 & 5 & $2,44 \pm 0,98$ & 10 & 1,97 & 0,86 \\
\hline lokpa & 45 & 1 & 5 & $2,27 \pm 0,82$ & 19 & 2,24 & 0,76 \\
\hline mahi & 25 & 1 & 5 & $2,32 \pm 1,18$ & 15 & 2,49 & 0,92 \\
\hline m'bèrmè & 11 & 2 & 4 & $2,81 \pm 0,75$ & 12 & 2,20 & 0,89 \\
\hline mina & 14 & 1 & 3 & $1,79 \pm 0,80$ & 6 & 1,60 & 0,89 \\
\hline mokolé & 8 & 1 & 5 & $2,13 \pm 1,36$ & 6 & 1,64 & 0,92 \\
\hline nago & 16 & 1 & 3 & $1,94 \pm 0,77$ & 14 & 2,46 & 0,93 \\
\hline peulh & 23 & 1 & 3 & $1,61 \pm 0,66$ & 11 & 2,12 & 0,88 \\
\hline sahouè & 27 & 1 & 5 & $1,81 \pm 0,96$ & 15 & 2,24 & 0,83 \\
\hline tchabè & 11 & 1 & 2 & $1,36 \pm 0,50$ & 5 & 1,31 & 0,82 \\
\hline waama & 20 & 1 & 4 & $2,35 \pm 0,67$ & 7 & 1,64 & 0,84 \\
\hline ouatchi & 17 & 1 & 3 & $1,47 \pm 0,71$ & 5 & 1,23 & 0,76 \\
\hline wémè & 38 & 1 & 3 & $1,89 \pm 0,66$ & 10 & 1,34 & 0,58 \\
\hline xhla & 7 & 1 & 2 & $1,71 \pm 0,49$ & 4 & 1,13 & 0,81 \\
\hline yoom & 14 & 2 & 5 & $2,71 \pm 0,99$ & 11 & 2,09 & 0,87 \\
\hline $\begin{array}{l}\text { Milieu } \\
\text { d'étude }\end{array}$ & 760 & 1 & 6 & $1,98 \pm 0,97$ & 387 & $1,97 \pm 0,58$ & $0,84 \pm 0,09$ \\
\hline
\end{tabular}




\section{Gnawe et al. J. Appl. Biosci. $2016 \quad$ Nomenclature vernaculaire et diversité des variétés locales des gombos (Abelmoschus spp.) cultivées au Bénin}

\section{DISCUSSION}

Cette étude a permis de recenser 24 noms vernaculaires pour désigner le mot gombo et 480 différentes appellations pour les variétés locales de gombos à travers les 31 ethnies explorées. Ceci constitue une contribution très importante à la documentation des différentes appellations pour les gombos cultivés au Bénin par rapport aux 51 appellations rapportées par Gnawé (2015) pour les variétés locales de gombos cultivées au sud-ouest du pays. Les résultats obtenus soulèvent le problème de la perception paysanne de l'hiérarchie taxonomique au niveau des unités systématiques en matière de la nomenclature vernaculaire des variétés locales de gombos cultivées. En effet, la nomenclature populaire des gombos ne fait aucune distinction entre les 3 espèces signalées au Bénin par Gnawé (2015) ; les variétés locales désignées étant toutes d'un même rang taxonomique et du même taxon selon la nomenclature locale. Les 24 noms attribués au mot gombos sont des génériques utilisés pour désigner indifféremment les espèces cultivées au Bénin. C'est ce qui expliquerait le fait que Akoègninou et al. (2006) et Dansi et al. (2008) ont rapporté 21 noms désignant l'espèce Abelmoschus esculentus alors que dans la réalité ces noms sont en fait des noms génériques pour désigner toute espèce de gombo. En considérant les 24 noms génériques attribués aux gombos dans les différentes langues locales, on s'aperçoit du rapprochement de certaines ethnies dérivant d'un même groupe sociolinguistique ou partageant la même aire socioculturelle (tableau 1). II s'agit par exemple des ethnies Ifè, Idaasha, Holli, Koura, Nagot et Tchabè dérivant toutes, sur le plan linguistiques, de l'ethnie Yoruba selon Amadou Sanni et Atodjinou (2012) et chez qui le gombo est partout appelé ila. De plus, ces ethnies rapprochées, partageant ou pas le même espace géographique de vie que d'autres ethnies, arrivent à garder leur authenticité linguistique en termes de désignation du gombo. L'espace géographique n'a donc pas influencé, sur le plan étymologique, les noms attribués au gombo par les diverses ethnies.

Les résultats ont aussi montrés que sur le plan taxonomique, la nomenclature vernaculaire des gombos cultivés repose sur l'utilisation des caractères morphologiques, agronomique et culturel. Parmi les 9

\section{CONCLUSION}

La présente étude a fait le premier point sur les variétés locales des gombos cultivés au Bénin. Au total, 480 appellations à travers les 31 ethnies enquêtées ont été recensées. Elles sont spécifiques aux groupes ethniques caractères utilisés par les producteurs enquêtés, 5 ont été signalés comme critères de taxonomie vernaculaire au Burkina Faso et en Colombie. Il s'agit de la couleur du péricarpe, la durée du cycle végétatif, la ressemblance du fruit aux organes d'animaux, la forme du fruit, l'origine géographique du taxon (Sadiki et al., 2007 ; Haoua et al., 2011). Les 4 critères restants tels que la valeur culinaire du fruit, les interdits alimentaires, la rugosité du péricarpe et la ressemblance du fruit à d'autres fruits constituent une spécificité aux enquêtés de la présente étude. Ces résultats confirment les travaux de Spichiger et al. (2000) qui ont affirmé que l'utilisation de caractères botaniques macroscopiques ou utilitaires dans la nomenclature et l'identification des espèces ne date pas de l'ère des scientifiques mais de l'antiquité et continue, à nos jours, d'être appliquée par les communautés locales. Les travaux de Sawadogo et Balma (2003) ont abouti à la conclusion que la nomenclature des variétés par les paysans repose sur des caractères agro-morphologiques facilement distincts. Sur le plan de la diversité, 387 variétés locales, considérées différentes les unes des autres suivant la perception paysanne, ont été recensées. Cette diversité est nettement au-delà de celle de 16 obtenue au Burkina Faso par (Haoua Jiro et al., 2011). Mais, le nombre de 387 variétés locales ne saurait exprimer la diversité variétale réelle des gombos cultivés au Bénin à cause des problèmes de synonymies et d'homonymies variétales entre différentes ethnies. Les valeurs de diversité variétale ne pourraient être proches de la réalité qu'à échelle des ethnies quand bien même les phénomènes de synonymie et d'homonymie sont possibles au sein d'une même ethnie comme l'ont déjà constaté les travaux de Dansi et al. (2008) au niveau des légumes feuilles traditionnels du Bénin. Le nombre de variétés locales détenues varie de 1 chez les boufalé à 41 chez les bariba. Les bariba constituent alors l'ethnie la plus détentrice de variétés locales au Bénin. Ceci améliore nettement les premiers inventaires variétaux de gombos qui ont rapporté 15 variétés produites dans le nord du Bénin par les bariba (Hamon et charrier, 1983). Ceci complète l'idée de Hamon et Charrier (1997) qui pensent le nombre de variétés de gombos dont dispose chaque région dépend de l'importance que l'ethnie principale accorde aux gombos.

qui ont gardé leur authenticité linguistique en termes de nomenclature des gombos. La nomenclature vernaculaire des variétés de gombos est de type binomial et repose sur des caractères agro-morphologiques. En prenant en 
compte les synonymies et les homonymies au niveau des noms au sein des ethnies respectives, les 480 appellations sont regroupées en 387 variétés locales. Mais, cette diversité variétale nationale n'est que le cumul des diversités ethniques. Elle pourrait regorger beaucoup de synonymies inter-éthiques et même d'autres

\section{REMERCIEMENT}

Nous remercions, la Fondation Internationale pour la Science (IFS) pour avoir octroyé la bourse de recherche

\section{RÉFÉRENCES BIBLIOGRAPHIE}

Achigan-Dako G. E., Pasquini W. M., Assogba-Komlan F., N'Danikou S., Yédomonhan H., Dansi A. et Ambrose-Oji B. (2010). Traditional vegetable in Benin. Institut National des Recherches Agricoles du Bénin. Imprimerie du CENAP, Cotonou, $286 \mathrm{p}$.

Akoègninou A., Van der Burg W.J., Van der Maesen L.J.G. (2006). Flore Analytique du Bénin. Backhuys Publishers, Leiden, Pays-Bas. 1034 p.

Amadou S., Atodjinou C. M. (2012). État et dynamique des langues nationales et de la langue française au Bénin. Québec : Observatoire démographique et statistique de l'espace francophone/Université Laval, (Collection Rapport de recherche de l'ODSEF), $54 \mathrm{p}$.

Borssum Waalkes J. Van (1966). Malesian Malvaceae revised. Blumea 14 (1) 1-151

Dansi A., Adjatin A., Adoukonou-Sagbadja H., Falade V., Yedomonhan H., Odou D. et Dossou B. (2008). Traditional leafy vegetables and their use in the Benin Republic. Genetic Resources and Crop Evolution 55: 1239-1256.

FAOSTAT. (2013). FAO Statistics, Food and Agriculture Organization of the United Nations, Rome. Based on data from the 2012 crop year downloaded October 2013 from: http://faostat.fao.org/site/567/default.aspx\#ancor 2011, Fiber Organon

Frontier S. \& Pichod-Viale D. (1995). Théorie des ecosystèmes: Structure fonctinnement, évolution, 2 è édition. Collection d'écologie 21 : 287-311. Paris, Masson.

Gnawé M. (2015). Diversité et ethnobotanique des gombos (Abelmoschusspp.) cultivés au Benin: cas des départements du Mono et du Couffo au Bénin, 52 p.

Hamon S. et Charrier A. (1983). Large variation of okra collected in Benin and Togo. Plant Genet. Res. Newsl 56: 52-58. synonymies et homonymies intra-éthiques non signalées par les producteurs. Ceci nécessite la caractérisation agro-morphologique de toutes les accessions collectées afin d'identifier les variétés ayant différentes appellations à travers les différentes ethnies et celles désignées anormalement sous le même nom vernaculaire.

$n^{\circ} \mathrm{C}$ 5595-1 qui a couvert les travaux de terrain entrant dans le cadre du présent article.

Hamon S. et Charrier, A. (1997). Les gombos. In: L'amélioration des plantes tropicales. Centre de coopération internationale en recherche agronomique pour le développement (CIRAD) et Institut français de recherche scientifique pour le développement en coopération (ORSTOM), Montpellier, France. pp. 313-333.

Haoua J., Sawadogo M. et Millogo J. (2011). Caractérisation agromorphologique et anatomique du gombo du Yatenga et leur lien avec la nomenclature locale des variétés. Sciences et Nature 8 (1): $23-36$

INSAE (2013). Recensement général de la population et de l'habitat, Résultats provisoires du RGPH4, MPPD, Cotonou, Bénin, 7p.

Nana R., Tamini Z., Sawadogo M. et Some P. P. (2010). Etude morphologique comparative de cinq variétés de gombo [Abelmoschus esculentus (L.) Moench] soumises à un stress hydrique. J. Sci. 10 (3): 28-38.

Sadiki M., Jarvis D., Rijal D., Bajrachrya J., Hue N. N., Camacho-villa T. C., Burgos-May L. A., Sawadogo M., Balma D., Lope D., Arios L., Mar I., Karamura D., Williams D., Chavez-Servia J. L., Shapit B., et Rao V. R. (2007). Variety names : An entry point of crop genetic diversity and distribution in agroecosystems. In Managing Bio-diversity in Agricultural Ecosystems. Biodiversity International, Jarvis DI (ed). Colombia University press: New York. 77-115 $\mathrm{pp}$.

Sawadogo M. et Balma D. (2003). Etude de la variabilité agromorphologique de quelques écotypes locaux de gombo cultivés au Burkina Faso. Science et Technique, Série Sciences Naturelles et Agronomie 27 (1-2): 111-129.

Siemonsma J. S. et Kouamé, C. (2004). Abelmoschus esculentus (L.) Moench Dans : Grubben, GJH \& Denton, OA (Editors). PROTA 2: Légumes I 
Gnawe et al. J. Appl. Biosci. $2016 \quad$ Nomenclature vernaculaire et diversité des variétés locales des gombos (Abelmoschus spp.) cultivées au Bénin

Légumes. [CD-Rom]. PROTA, Wageningen, Pays-Bas.

Spichiger R-E., Savolainen V. V., Figeat M. (2000). Botanique systématique des plantes: Une approche phylogénétique nouvelle des Angiospermes des régions tempérées et tropicales. Première édition. $373 p$ 